\title{
Vinculación con la sociedad en la formación militar de la carrera en Ciencias Aeronáuticas Militares
}

\section{Association with society in the military training of the career in Military Aeronautical Sciences}

\author{
César Quinga Suárez ${ }^{1 *}$ \\ ${ }^{1}$ Universidad de Fuerzas Armadas - ESPE, Ecuador \\ *cesarquin0906@yahoo.com \\ DOI: https://doi.org/10.26871/killkanasocial.v4i2.722
}

\begin{abstract}
Resumen
Las actividades de vinculación con la sociedad y de acuerdo con la Ley Orgánica Reformatoria a la Ley Orgánica de Educación Superior 2018, en el que se establece el cumplimiento de la docencia, investigación e innovación y vinculación con la sociedad, la carrera en Ciencias Aeronáuticas Miliares desarrolla el proyecto de vinculación para el uso y manejo de las nuevas herramientas tecnológicas de la información y comunicación (NTIC), con el objetivo de establecer la prevención y seguridad infantil en el uso de redes sociales, en la población urbano marginal de la zona de planificación José Luis Tamayo, parroquia de Salinas, provincia de Santa Elena, en el que se encuentra la Escuela de Educación Básica “Francisco Huerta Rendón, con una capacidad de 700 estudiantes. De igual manera el proyecto se relaciona con la línea de investigación de la carrera de Seguridad y Defensa; conjuntamente con los objetivos del Plan Nacional Del Buen Vivir y los objetivos estratégicos que se encontraban vigentes en el 2016, con el propósito de usar las herramientas tecnológicas y mejorar la calidad del aprendizaje, brindando a los estudiantes la oportunidad de avanzar en las destrezas para el tratamiento de la información que se requiere en la formación académica. Finalmente, el proyecto que se desarrollo tuvo impacto de manera directa en la población estudiantil y planta docente de la institución educativa, así como en el entorno familiar.
\end{abstract}

Palabras clave: vinculación, seguridad, defensa, herramientas, tecnológicas.

\begin{abstract}
The linkage-related activities with society and in accordance with the Organic Law Reform to the Organic Law of Higher Education 2018, which establishes the fulfillment of teaching, research and innovation and links with society, the career in Military Aeronautical Sciences develops the linkage project for the use and management of the new information and communication technologies, with the aim of establishing prevention and child safety in the use of social networks, in the marginal urban population in the planning area José Luis Tamayo, parish of Salinas, province of Santa Elena, which is the School of Basic Education "Francisco Huerta Rendón, with a capacity of 700 students. Likewise, the project is related to the research line of the Security and Defense career; together with the objectives of the National Plan of Good Living and the strategic objectives that were applicable in 2016, with the purpose of using technological tools and improving the quality of learning, offering students the opportunity to advance in the skills for treatment of the information that is required in the academic formation. Finally, the project that was developed and it had a direct impact on the student population and teaching staff of the educational institution, as well as on the family environment.
\end{abstract}

Keywords: bonding, security, defense, tools, technological.

\section{Introducción}

El proyecto de vinculación fue desarrollado en el 2016, por parte de los cadetes de cuarto año de la Escuela Superior Militar de Aviación “Cosme Rennella B.”, con el título, Uso y manejo de las nuevas herramientas tecnológicas de la información y comunicación (NTIC) en la prevención y seguridad infantil en el uso de redes sociales, para la población urbano marginal de la zona de planificación José Luis Tamayo del Cantón Salinas, en el que se identificó que no cuenta con el conocimiento necesario de la seguridad frente al acoso infantil poniendo en peligro vida personal y la de su familia.

El crecimiento constante del aspecto tecnológico en todos los ámbitos de la sociedad, y en especial en redes sociales, ha determinado que las familias, docentes y autoridades educativas no puedan mantener el control directo en los estudiantes. 
El proyecto se desarrolló cumpliendo la programación de 90 horas de duración, dirigido a 993 estudiantes que obtuvieron conocimiento en el uso de las nuevas herramientas tecnológicas de la información y comunicación, minimizando el impacto en el uso de redes sociales.

Del planteamiento anterior, el $100 \%$ de estudiantes de la escuela Francisco Huerta Rendón, y la totalidad de los padres de familia desconocen del acoso infantil por medios informáticos en especial en las redes sociales, conjuntamente con la existencia de 15 docentes que poseen escaso conocimiento en la temática tratada.

Entre los problemas que presentan los adolescentes por desconocimiento de las redes sociales (Aftab, 2006), se encuentran el cyberbullying, bullyingque, grooming, considerados como amenazas, acosos, humillaciones, situaciones vergonzosas, que se convirtien en el blanco ideal para extorciones a través de herramientas tecnologías interactivas, digitales o teléfonos móviles.

En el mismo orden de ideas (Garaigordobil, 2011) considera: "La Comisión Europea, en su Programa Safe Internet para el período 2009-2013, considera el Cyberbullying (CB) como una amenaza relevante en la infancia y la adolescencia tanto por su alto nivel de prevalencia por la gravedad de sus consecuencias" (p.235).

A este planteamiento la tecnología se ha convertido un medio para acceder a un mundo de la comunicación, a través de la conectividad y complejidad como parte de las estrategias educativas, originando una visión en el entorno académico indispensable para poder interactuar entre los estudiantes y docentes, pero al mismo tiempo este criterio permite relacionarse en las redes sociales y es allí donde se presente el acoso directo a los niños y adolescentes, razón por la cual se desarrolla el proyecto de vinculación con la sociedad que los cadetes de cuarto año militar se relacionan con las comunidades que necesitan conocer de los nuevos patrones de comportamiento en el aspecto tecnológico y así minimizar el impacto que se presenta mediante el rastreo de terceras personas.

\section{Marco teórico}

\subsection{Redes Sociales}

En los actuales momentos las redes sociales representan un servicio público que articulan en un entorno social mediante las conexiones tecnológicas (Alonso y Bartolomé, 2014), como parte de las herramientas informáticas en la que permite cambiar las ideas y sentimientos del contexto dentro de una sociedad globalizada.

De la misma manera, se conllevan información desde varias perspectivas como entes de la sociedad, por otro lado, se encuentran los estudiantes que forman comunidades o grupos académicos para compartir y debatir ciertos tópicos que se plantean, sin dejar de lado los individuos que simplemente comparte datos personales con diferentes fines, e allí el uso correcto de las redes sociales.

De acuerdo con (Ortega y Aguillo, 2008) las redes sociales "se ha convertido en un medio de creación cola- borativa en la que usuarios particulares, de forma libre y gratuita, aportan experiencias" (p.603)

\subsection{Cyberbullying}

Según el planteamiento de (Aquino, 2014), el Cyberbullying "es una agresión repetitiva de uno o más individuos en contra de una persona, utilizando dispositivos o servicios de tecnología" (p.1), a diferencia de la persecución tradicional conocido con el bullying, este nuevo tema está presente mediante las redes sociales, en donde los actores del acoso puede ser una o varias personas que realizan ataques de divulgación de información personal de la víctima o datos falsos que se genera con el propósito de acosar al individuo.

El acoso se genera mediante las TIC, por medio de los celulares, redes sociales, foros, chat, blog, juegos interactivos $\mathrm{u}$ otra herramienta tecnológica, además de generar perfiles falso, lo cual da lugar a la creación de daños psicológicos en la victima, para poder evitar esto se tiene que concientizar a la población el uso restringido y sobre todo estar presente toda la comunidad educativa de maneta activa en este tema de acoso por la red.

\subsection{Bullying}

El bullying o también conocido como acoso en el que se genera agresión física o verbal, además de actos hostiles, (Ruiz, 2011) "se refiere a todas las formas de actitudes agresivas, intencionadas y repetidas, que ocurren sin motivación evidente, adoptadas por uno o más estudiantes contra otro u otros, lo hace para imponer su poder, a través de constantes amenazas, insultos, agresiones, vejaciones, y así tenerlo bajo su completo dominio a lo largo de meses e incluso años" (p.21).

Este tipo de acoso genera cambios de la conducta en la victima, desequilibrio en el ejercicio de las actividades, variación en los valores sociales, además de las relaciones interpersonal en los centros educativos en donde se generan secuelas que se reflejan variaciones en el comportamiento de los individuos tanto en el hogar como en los establecimientos académicos, lo que origina agresiones y conflictos constantes, (Ceron y Uberti, 2017).

\subsection{Grooming}

Según (Palmer, 2017) se define como "el ciberacoso ejercido de forma deliberada por un adulto para establecer una relación y un control emocional sobre un menor con el fin de preparar el terreno para su abuso sexual" (p.27), generalmente se desarrolla cuando la persona acosadora es mayor que la víctima, inicia el proceso con la confianza y la obtención de cierta información personalizada y pormenorizada, entre los que están: la edad, gustos, lugares en los que frecuenta, inclusive de fotos, videos, lo que genera un intereses mutuos con la finalidad de abusos sexuales, luego de un tiempo serán utilizados en beneficio personal, con el fin de hacer daño al individuo indefenso. 
Igual que los aspectos analizados anteriormente se originan daños psicológicos, depresión, baja autoestima, desconfianza, por otro lado, se incrementa el mal humor, disminuye el rendimiento académico, en fin las afectaciones cada vez se van incrementando conforme el acosador utiliza los datos obtenida de la víctima, el cual incluye una extorción económica para no publicar la información respectiva.

\section{Metodología}

El proyecto tuvo una duración de 108 horas clasificadas de la siguiente manera 90 horas desarrolladas en la
Unidad Educativa "Francisco Huerta Rendón” y 18 horas en los Barrios Bazán y Coresa con un número de beneficiarios directos de 1827 y 2032 indirectos.

\subsection{Involucrados}

Fechas inicio: 18 de marzo del 2016

Fechas de finalización: 10 de octubre del 2016

1) Escuela Superior Militar de Aviación

2) Dirección Nacional de Policía, Especializada para niños, niñas y adolecentes

3) Barrios Coresa1 y Bazán

4) Unidad Educativa "Francisco Huerta Rendón"

Tabla 1: Barrios Coresa 1 y Bazán

\begin{tabular}{|c|c|c|c|}
\hline Actividades & Resultados esperados & $\begin{array}{c}\% \\
\text { Cumplimiento }\end{array}$ & Observaciones \\
\hline $\begin{array}{l}\text { Firma de Acta de Compromiso por par- } \\
\text { te de los beneficiares de los Barrio Co- } \\
\text { resa } 1 \text { y Bazán }\end{array}$ & $\begin{array}{l}\text { Compromiso de los } 2 \text { barrios por } \\
\text { parte de los presidentes barriales. }\end{array}$ & $100 \%$ & $\begin{array}{l}\text { Barrios Involucrados Core- } \\
\text { sa } 1 \text { y Bazán }\end{array}$ \\
\hline $\begin{array}{l}\text { Difusión del proyecto mediante la so- } \\
\text { cialización en las radiodifusoras de la } \\
\text { península }\end{array}$ & $\begin{array}{l}\text { Socialización a la población de la } \\
\text { península específicamente al cantón } \\
\text { salinas sobre las amenazas del uso } \\
\text { de las nuevas tecnologías en la ni- } \\
\text { ñez y adolescencia. }\end{array}$ & $100 \%$ & $\begin{array}{l}\text { Se realizaron } 2 \text { entrevistas } \\
\text { en la Radio Amor y Antena } \\
3\end{array}$ \\
\hline Charla a los barrios involucrados & $\begin{array}{l}\text { Comprometimiento de los habitan- } \\
\text { tes de los barrios involucrados en el } \\
\text { proyecto. }\end{array}$ & $100 \%$ & Barrio Bazán Barrio Coresa \\
\hline $\begin{array}{l}\text { Inducción sobre el proyecto a los ba- } \\
\text { rrios involucrados. }\end{array}$ & $\begin{array}{l}\text { Conocimiento a los habitantes de } \\
\text { los barrios involucrados en el pro- } \\
\text { yecto acerca de los objetivos y la } \\
\text { problemática que se pretende sol- } \\
\text { ventar. }\end{array}$ & $100 \%$ & Barrio Bazán Barrio Coresa \\
\hline $\begin{array}{l}\text { Conferencia a los barrios involucrados } \\
\text { sobre Generalidades y Conocimiento } \\
\text { básicos }\end{array}$ & $\begin{array}{l}\text { Conocimientos básicos adquiridos } \\
\text { sobre las NTIC. }\end{array}$ & $100 \%$ & Barrio Bazán Barrio Coresa \\
\hline $\begin{array}{l}\text { Conferencia a los barrios involucrados } \\
\text { sobre sistemas de seguridad y recomen- } \\
\text { daciones para el uso de nuevas tecnolo- } \\
\text { gías }\end{array}$ & $\begin{array}{l}\text { Conocimientos sobre el correcto } \\
\text { uso y manejo de las NTIC orientado } \\
\text { a su labor diario. }\end{array}$ & $100 \%$ & Barrio Bazán Barrio Coresa \\
\hline $\begin{array}{l}\text { Conferencia a los barrios involucrados } \\
\text { sobre Nuevas tecnologías y seguridad }\end{array}$ & $\begin{array}{l}\text { Conocimiento enfocado al resguar- } \\
\text { do de información personal debido } \\
\text { al suplanta miento de identidades. }\end{array}$ & $100 \%$ & Barrio Bazán Barrio Coresa \\
\hline
\end{tabular}

Tabla 2: Unidad educativa "Francisco Huerta Rendón"

\begin{tabular}{|c|c|c|c|}
\hline Actividades & Resultados esperados & $\begin{array}{c}\% \\
\text { Cumplimiento }\end{array}$ & Observaciones \\
\hline $\begin{array}{l}\text { Inauguración del proyecto y firma del } \\
\text { acta de compromiso como institución } \\
\text { beneficiaria }\end{array}$ & $\begin{array}{l}\text { Presentación del proyecto de vincu- } \\
\text { lación a través de la participación } \\
\text { de los } 38 \text { cadetes a los beneficiarios } \\
\text { de la comunidad educativa. }\end{array}$ & $100 \%$ & $\begin{array}{l}\text { Presencia de } 240 \text { de asisten- } \\
\text { tes: (autoridades militares y } \\
\text { educativas, beneficiarios di- } \\
\text { rectos) }\end{array}$ \\
\hline $\begin{array}{l}\text { Inducción a las nuevas tecnologías y } \\
\text { herramientas de comunicación }\end{array}$ & $\begin{array}{l}\text { Conocimientos básicos sobre las } \\
\text { nuevas herramientas tecnológicas } \\
\text { que existen en la actualidad. }\end{array}$ & $100 \%$ & $\begin{array}{l}\text { Beneficiarios: Estudiantes y } \\
\text { docentes }\end{array}$ \\
\hline
\end{tabular}


Tabla 2: Unidad educativa "Francisco Huerta Rendón"...(continuación)

\begin{tabular}{|c|c|c|c|}
\hline Actividades & Resultados esperados & $\begin{array}{c}\% \\
\text { Cumplimiento }\end{array}$ & Observaciones \\
\hline $\begin{array}{l}\text { Conferencia sobre ventajas y desventa- } \\
\text { jas del avance tecnológico }\end{array}$ & $\begin{array}{l}\text { Conocimientos básicos sobre las } \\
\text { ventajas y desventajas de los avan- } \\
\text { ces tecnológicos que actualmente } \\
\text { en nos brinda la tecnología a través } \\
\text { del uso de las redes sociales. }\end{array}$ & $100 \%$ & $\begin{array}{l}\text { Beneficiarios: Estudiantes y } \\
\text { docentes de la Unidad }\end{array}$ \\
\hline $\begin{array}{l}\text { Conferencia sobre Redes sociales en } \\
\text { Internet y del Cyberbullying, Bullying- } \\
\text { que, Grooming, }\end{array}$ & $\begin{array}{l}\text { Conocimiento general sobre el tipo } \\
\text { de redes sociales más frecuentadas } \\
\text { por la juventud, su uso y manejo. }\end{array}$ & $100 \%$ & $\begin{array}{l}\text { Beneficiarios: Estudiantes y } \\
\text { docentes de la Unidad }\end{array}$ \\
\hline $\begin{array}{l}\text { Conferencia sobre Identidad digital en } \\
\text { la Conectividad }\end{array}$ & $\begin{array}{l}\text { Conocimiento sobre el manejo y } \\
\text { gestión de la identidad digital y de } \\
\text { información personal dentro las re- } \\
\text { des sociales. }\end{array}$ & $100 \%$ & $\begin{array}{l}\text { Beneficiarios: Estudiantes y } \\
\text { docentes de la Unidad }\end{array}$ \\
\hline $\begin{array}{l}\text { Conferencia sobre Configuración de se- } \\
\text { guridad y privacidad en redes sociales }\end{array}$ & $\begin{array}{l}\text { Conocimiento sobre las restriccio- } \\
\text { nes, configuraciones de privacidad, } \\
\text { términos de condiciones y uso en el } \\
\text { manejo de las redes sociales. }\end{array}$ & $100 \%$ & $\begin{array}{l}\text { Beneficiarios: Estudiantes y } \\
\text { docentes de la Unidad }\end{array}$ \\
\hline $\begin{array}{l}\text { Conferencia sobre Fenómenos en redes } \\
\text { sociales y sus consecuencias }\end{array}$ & $\begin{array}{l}\text { Que los estudiantes sepan proteger } \\
\text { su privacidad e información perso- } \\
\text { nal en las redes sociales. }\end{array}$ & $100 \%$ & $\begin{array}{l}\text { Beneficiarios: Estudiantes y } \\
\text { docentes de la Unidad }\end{array}$ \\
\hline $\begin{array}{l}\text { Conferencia para padres y Comunidad } \\
\text { Educativa }\end{array}$ & $\begin{array}{l}\text { Uso de las herramientas de informa- } \\
\text { ción y comunicación que los niños } \\
\text { utilizan y que los padres deben co- } \\
\text { nocer. }\end{array}$ & $100 \%$ & $\begin{array}{l}\text { Beneficiarios: Estudiantes, } \\
\text { docentes y comunidad aca- } \\
\text { démica de la Unidad }\end{array}$ \\
\hline $\begin{array}{l}\text { Conferencia sobre una guía legal para el } \\
\text { uso de internet }\end{array}$ & $\begin{array}{l}\text { Conocimientos elementales sobre } \\
\text { una guía legal para el uso de inter- } \\
\text { net derecho de autor de la informa- } \\
\text { ción. }\end{array}$ & $100 \%$ & $\begin{array}{l}\text { Beneficiarios: Estudiantes y } \\
\text { docentes de la Unidad }\end{array}$ \\
\hline $\begin{array}{l}\text { Actividades de recreación contra la } \\
\text { tecno-adicción }\end{array}$ & $\begin{array}{l}\text { Realización de actividades físicas, } \\
\text { sociales, culturales que aporten a } \\
\text { los niños a poner interés en este } \\
\text { tipo de dinámicas para evitar las } \\
\text { adicciones en las redes sociales. }\end{array}$ & $100 \%$ & $\begin{array}{l}\text { Beneficiarios: Estudiantes } \\
\text { de la Unidad }\end{array}$ \\
\hline Clausura del proyecto de vinculación & $\begin{array}{l}\text { Culminación del proyecto de vincu- } \\
\text { lación a través de la participación } \\
\text { de los } 38 \text { cadetes a los beneficiarios } \\
\text { de la comunidad educativa. }\end{array}$ & $100 \%$ & $\begin{array}{l}\text { Beneficiarios: Estudiantes y } \\
\text { docentes de la Unidad }\end{array}$ \\
\hline
\end{tabular}

Tabla 3: Cumplimiento de objetivos y resultados

\begin{tabular}{ll}
\hline \multicolumn{1}{c}{ Objetivos } & \multicolumn{1}{c}{ Resultados } \\
\hline $\begin{array}{l}\text { Socializar los nuevos elementos teóricos -metodológicos } \\
\text { para el uso y aprovechamiento de la informática en la } \\
\text { prevención y seguridad frente al acoso infantil }\end{array}$ & $\begin{array}{l}\text { Se capacitaran 15 docentes en 90 horas y se entregaran } \\
\text { folles digitales e impresos para que sean utilizados como } \\
\text { guía de enseñanza. }\end{array}$ \\
$\begin{array}{l}\text { Desarrollar en la comunidad educativa conformada por 993 } \\
\text { personas destrezas aplicadas a la informática y pensamiento } \\
\text { crítico. }\end{array}$ & $\begin{array}{l}\mathbf{4 4 3} \text { Estudiantes, } \\
\mathbf{1 5} \text { Docentes, } \\
\end{array}$ \\
$\mathbf{1 0 5 7}$ Padres de familia \\
Beneficiarios directos (Unidad Educativa)
\end{tabular}


Tabla 3: Cumplimiento de objetivos y resultados...(continuación)

\begin{tabular}{ll}
\hline Objetivos & Resultados \\
\hline
\end{tabular}

138 Barrio Bazán

3859 Total Favorecidas

Con el proyecto de vinculación con la sociedad que forman parte de los recursos educativos para prevención y seguridad infantil en el uso de redes sociales.

Asegurar el desarrollo del campo informático y la interacción entre estudiantes y docentes.
El $100 \%$ de los docentes se comprometieron a realizar clases periódicas para la actualización y refrescamiento de los conocimientos adquiridos.

\section{Resultados}

Comparación de la situación inicial con la actual de la población luego de la aplicación del proyecto de vinculación con la sociedad:

La Unidad Educativa "Francisco Huerta Rendón" conformado por los padres de familia, estudiantes, docentes y autoridades del plantel recibieron capacitaciones del buen uso y manejo de las NTIC de la información, en las que se evidenciaron algunas situaciones actuales de cada uno de los beneficiarios y que se desconocía sobre el mal uso de estas herramientas tecnológicas en el acceso a datos personales y la utilización inadecuada de estos medios. Los padres de familia con este tipo de información se creó una cultura de concientización que permita el funcionamiento de la seguridad de sus hijos; ya que algunos desconocían de los riesgos y peligros que están acechos y propensos los niños en las redes sociales.

Por otro lado, en los estudiantes, se concientizo los peligros, riesgos y las consecuencias del uso de las redes sociales que están expuestos en el manejo y buen uso de la información y en el correcto uso de los medios de información y comunicación.

Además, los docentes y autoridades comprendieron de las graves consecuencias que representa el mal uso de las redes sociales y sobre todo de la situación socio-educativa del entorno de la población beneficiaria.

\subsection{Grado de impacto del proyecto en la comunidad}

El grado de impacto del proyecto es alto; por el eficaz cumplimento de los objetivos planteados evidenciados en los registros de asistencia de los beneficiarios alumnos, padres de familia, docentes y autoridades de la Escuela de educación básica "Francisco Huerta Rendón"

Además, se sumaron al proyecto los barrios: Bazán y Coresa 1, incrementando el número total de beneficiarios, los mismos que desarrollaron nuevas actitudes y aptitudes de prevención y seguridad informática.

4.2 Grado de impacto del proyecto en las actividades de vinculación con la sociedad del departamento

El desarrollo del proyecto marca un precedente positivo en el Departamento de Seguridad y Defensa, con base en las experiencias y vivencias de los estudiantes y docentes participantes, que coadyuvan para la realización y planificación de futuros proyectos con la sociedad, orientado a incrementar el número de beneficiarios directos e indirectos, para la oportuna diseminación del conocimiento y promoviendo la imagen de la institución.

\subsection{Grado de impacto del proyecto en actividades de formación del estudiante}

El estudiante-cadete reforzó las aptitudes encaminadas al desarrollo de las competencias genéricas del futuro oficial. Instruyendo y apoyando con conocimientos para contribución del cumplimiento de los objetivos encomendados a las FFAA en el Plan Nacional el Buen Vivir en el 2016.

\section{Conclusiones}

Participación de cuatro docentes de la Universidad de las Fuerzas Armadas - ESPE, los cuales supervisaron el desarrollo y planificación de las actividades programadas en el proyecto de vinculación.

Se capacitaron a los estudiantes en la carrera en la Licenciatura en Ciencias Aeronáuticas Militares en aspectos legales y prevención en el abuso de la tecnología en la niñez y adolescencia por un periodo de 20 horas.

La comunidad beneficiaria señalo sentirse respaldada y segura, ya que pueden enfrentar cualquier eventualidad y con el compromiso de seguir impartiendo y difundiendo en su entorno los conocimientos adquiridos durante las capacitaciones brindas por los cadetes-estudiantes.

\section{Recomendaciones}

Promover la cooperación interinstitucional para continuar ejecutando proyectos en bien de la comunidad y mitigar las amenazas existentes en el entorno de la comunidad académica.

Mantener un vínculo constante con la comunidad para proponer nuevos temas de interés común para que la sociedad se sienta respaldada con nuestra institución. 


\section{Referencias Bibliográficas}

Aftab, P. (2006). Ciberbullying. Guía práctica para madres, padres y personal docente. Bilbao: Fundación EDEX.

Alonso, M., y Bartolomé, A. (2014). Redes sociales y consumidores: participación y construcción de imagen. Historia y Comunicación Social, 211-221.

Aquino, R. (2014). Cyberbullying: acoso utilizando medios electrónicos. Revista Digital Universitaria, 1-8.

Ceron, M., y Uberti, L. (2017). Bullying en la escuela: inquiriendo las razones promotoras de losconflictos "entre" y "de los" alumnos. Buenos Aires: CLACSON.

Garaigordobil, M. (2011). Prevalencia y consecuencias del cyberbullying: una revisión. International Journal of Psychology and Psychological Therapy, 233-254.

Ortega, L., y Aguillo, I. (2008). Análisis estructural de una red social en línea:. El profesional de la información, 603,610 .

Palmer, F. (2017). Seguridad y Riesgos: Cyberbullying. Universidad Autonoma de Barcelona.

Ruiz, Y. (2011). El bullying en las escuelas. Revista Digital, $1-7$.

Recibido: 28 de abril de 2021

Aceptado: 5 de julio de 2020

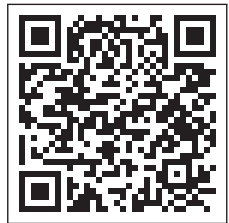

